\title{
Current and emerging therapy for the management of vitiligo
}

\author{
Alicia Cecile Borderé \\ Jo Lambert \\ Nanny van Geel \\ University Hospital of Ghent, \\ Department of Dermatology, \\ Ghent, Belgium
}

Correspondence: Nanny van Geel Department of Dermatology, De Pintelaan 185, 9000 Ghent, Belgium Tel +3293322287

Fax +32 93324996

Email nanny.vangeel@ugent.be

\begin{abstract}
Vitiligo is an acquired cutaneous disorder of pigmentation, with an incidence of $0.5 \%$ to $2 \%$ worldwide. There are three major hypotheses for the pathogenesis of vitiligo that are not exclusive of each other: biochemical/cytotoxic, neural and autoimmune. Recent data provide strong evidence supporting an autoimmune pathogenesis of vitiligo. As vitiligo can have a major effect on quality of life, treatment can be considered and should preferably begin early when the disease is active. Current treatment modalities are directed towards stopping progression of the disease and achieving repigmentation. Therapies include corticosteroids, topical immunomodulators, photo(chemo)therapy, surgery, combination therapies and depigmentation of normally pigmented skin. Topical class 3 corticosteroids can be used for localized vitiligo. The use of topical immunomodulators (TIMs) in vitiligo seems to be equally effective as topical steroids, especially when used in the face and neck region. In photo(chemo)therapy, narrowband ultraviolet-B therapy (NB-UVB) seems to be superior to psoralen ultraviolet-A therapy (PUVA) and broadband UVB. In surgical techniques, split-thickness grafting and epidermal blister grafting were shown to be effective methods, although the non-cultured epidermal suspension technique has many advantages and seems to be a promising development. Depigmentation therapy can be considered if vitiligo affects more than $60 \%$ to $80 \%$ of the body. Complementary therapies such as Polypodium leucotomos show promising results in combination with UVB therapy. No causative treatment for vitiligo is currently available. More randomized controlled trials on the treatment of vitiligo are necessary.
\end{abstract}

Keywords: vitiligo, non surgical treatment, surgical treatment

\section{Introduction}

Vitiligo is an acquired cutaneous disorder of pigmentation, with a $0.5 \%$ to $2 \%$ incidence worldwide, without predilection for sex or race. Besides skin, oral mucosa and hair may also be depigmented. Classically, vitiligo is divided into segmental and generalized forms. The age of onset of vitiligo is variable, but peaks in the second and third decades. The depigmentation has a predilection for acral areas and around body orifices (mouth, eyes, nose, anogenital region). ${ }^{1}$

The Vitiligo European Task Force (VETF) was founded in 2003 during the ESPCR meeting in Ghent and formulated the following definition:

"Generalized vitiligo or non-segmental vitiligo (NSV) is an acquired chronic pigmentation disorder characterized by white patches, often symmetrical, which usually increase in size with time, corresponding to a substantial loss of functioning epidermal and sometimes hair follicle melanocytes".2

"Segmental vitiligo is an acquired chronic pigmentation disorder characterized by white patches with a unilateral distribution that may totally or partially match a dermatome, but not necessarily. Other distribution patterns can be encountered that cross several dermatomes, or correspond to large areas delineated by Blaschko's lines". 2 


\section{Pathogenesis}

The precise cause of vitiligo is unknown. There are 3 major hypotheses for the pathogenesis of vitiligo that are not exclusive of each other: biochemical/cytotoxic, neural and autoimmune. The autoimmune hypothesis is based on genetic data and the fact that vitiligo is associated with other autoimmune diseases. The cytotoxic theory postulates that cell death of melanocytes is caused by cytotoxic precursors to melanin synthesis. The neural hypothesis is based on case reports were patients with nerve injury develop vitiligo at the affected sites and links segmental vitiligo with neurons that interact with melanocytes and release melanocytotoxic substrates. ${ }^{3}$

As none of the three hypotheses is sufficient to explain fully the mechanisms of vitiligo, the convergence theory is proposed. This theory states that stress, accumulation of toxic compounds, infection, autoimmunity, mutations, altered cellular environment and impaired melanocyte migration and proliferation can all contribute in varying proportions to the etiopathogenesis of vitiligo. ${ }^{4}$

There is increasing evidence for an autoimmune pathogenesis of vitiligo. In 2000, van den Wijngaard et al analyzed lesional, perilesional, and non-lesional skin biopsies from patients with vitiligo and compared these with immune infiltrates found in the skin of normal healthy donors and relevant disease controls and found a major role for skin-homing $\mathrm{T}$ cells in the death of melanocytes seen in vitiligo. ${ }^{5}$

An autoimmune process is also suggested by the concomitant occurrence of other autoimmune diseases like autoimmune thyroiditis, diabetes mellitus, pernicious anemia, Addison's disease, primary adrenal insufficiency and hypopituitarism in patients with vitiligo. ${ }^{6}$

Genetic factors also appear to play a role in the etiopathogenesis of vitiligo as $20 \%$ to $30 \%$ of patients have a family history of the disorder. ${ }^{7}$ Vitiligo does not follow a normal Mendelian pattern of inheritance, and it has been suggested that the disease most likely has a multifactorial, polygenic basis. ${ }^{8}$

However, many patients with vitiligo have neither a family history of vitiligo nor a history of other autoimmune diseases. A number of other hypotheses have been proposed to explain the pathogenesis of this disorder, like an intrinsic abnormality of melanocytes, increased local catecholamine release, hypoxia, oxidative stress and melanocytorrhagia. ${ }^{9}$

\section{Treatment}

Since a causative treatment is not available, current treatment modalities are directed towards stopping the progression of vitiligo and achieving repigmentation in order to repair the morphology and functional deficiencies of the depigmented skin areas. ${ }^{10}$ Current treatment modalities aim to stimulate melanocyte proliferation or interfere with inflammatory factors affecting melanocyte structure or function. No single treatment method has yet been found that is consistently effective with relatively few side-effects. ${ }^{11}$

Current repigmentation therapies include corticosteroids, topical immunomodulators, photo(chemo)therapy, surgery and depigmentation of normally pigmented skin.

\section{Non-surgical repigmentation treatment Corticosteroids}

Different classes of topical corticosteroids were studied. Topical steroids are most effective for lesions on the face, elbows and knees, the distal extremities respond poorly. Factors contributing to the variation in repigmentation rate by anatomic site include skin permeability, migration of residual melanocytes from uninvolved skin, reversibility of melanocyte damage and especially preservation and density of follicular reservoirs. ${ }^{13}$

In 1977, Bleehen performed a placebo controlled study and treated 20 patients with vitiligo either with $0.1 \%$ betamethasone valerate or with $0.05 \%$ clobetasol propionate creams and similar control areas with placebo preparations and concluded that both topical corticosteroids could be used for the treatment of selected patients with vitiligo and can induce repigmentation of the skin. ${ }^{14}$ Clayton conducted a double blind study and compared $0,05 \%$ clobetasol proprionate in a cream base with the cream base alone and came to the conclusion that the active product was significantly superior to the cream base alone in the treatment of vitiligo. ${ }^{15}$

In a controlled randomized trial by Kandil, $0.1 \%$ betamethasone valerate in 50\% isopropyl alcohol was compared to the alcohol base alone in 19 patients with vitiligo. A higher percentage of lesions had complete repigmentation when treated with the active product as compared to placebo. ${ }^{16}$

A study by Lepe et al compared (left-right) $0.05 \%$ clobetasol proprionate with $0.1 \%$ tacrolimus for the treatment of vitiligo in children and showed a similar repigmentation rate of $49.3 \%$ for clobetasol and $41.3 \%$ for tacrolimus. ${ }^{17}$ Sanclemente et al compared the effect of topical $0.05 \%$ betamethasone valerate versus catalase/dismutase superoxide (C/DSO) in a randomized, matched-paired, double-blind trial with 25 patients and concluded that vitiligo repigmentation with topical C/DSO at 10 months is similar to repigmentation with topical $0.05 \%$ betamethasone valerate. ${ }^{18}$

In 1999, Njoo et al performed a meta-analysis of 20 randomized controlled trials and 75 patient series on the treatment 
of vitiligo and concluded that topical class 3 corticosteroids and ultraviolet-B therapy (UVB therapy) are the most effective and safest therapies for localized and for generalized vitiligo, respectively. ${ }^{19}$

The biggest side-effect of topical corticosteroids is skin atrophy, more seen in class 4 than in class 3 corticosteroids. Other side-effects were striae, hypertrichosis, acneiform eruption and telangiectasia.

High and low doses of oral corticosteroids in pulse therapy have been shown to be successful in patients with progressive disease..$^{20,21}$ Recently, Rath et al compared different phototherapy methods (psoralen ultraviolet-A PUVA [therapy], narrowband UVB and broadband UVB) with an oral minipulse of steroids (OMP) as an adjunct to determine the method with the best tolerability and efficacy. They randomly assigned 68 patients with progressive vitiligo to different study groups (OMP, OMP + PUVA, OMP + narrowband UVB, OMP + broadband UVB). Each patient was followed up for 6 months and then the treatment was discontinued. Clinical evaluation was made at the end of 3 and 6 months. They concluded that the oral steroids only had an adjunct value and were not very effective by themselves. Narrowband UVB is preferred over broadband UVB. Narrowband UVB and PUVA showed comparable efficacy. ${ }^{22}$

No placebo controlled studies have been performed for the use of oral corticosteroids in vitiligo yet. So their use is still considered controversial since systemic side-effects (eg, moon face, weight gain) are associated with this therapy.

\section{Photo(chemo)therapy}

The ultraviolet (UV)-spectrum is divided into 3 major groups: UVA, UVB en UVC. UVC-radiation (200-280 nm) is filtered by the atmosphere and has no place in phototherapy. UVBradiation $(280-320 \mathrm{~nm})$ is biologically the most active. UVA-radiation (320-400 nm) has the longest wavelength of the UV-spectrum and is biologically less active than UVBradiation. However, it is partly responsible for erythema and pigmentation induced by sunlight. ${ }^{23}$

\section{(P)UVA}

Only a few studies (no randomized, controlled trials) have been performed, studying UVA-therapy in vitiligo. El Mofty et al studied the effect of broadband UVA on vitiligo lesions in 20 patients, $15 \mathrm{~J} / \mathrm{cm}^{2}$ in group I and $5 \mathrm{~J} / \mathrm{cm}^{2}$ in group II, a total of 48 sessions over 16 weeks. All patients received 3-weekly sessions of UVA. Overall pigmentation of $60 \%$ and above was recorded in $50 \%$ and $10 \%$ of patients in groups I and II, respectively. They concluded that broadband UVA alone, without psoralens, may have an important therapeutic value in the treatment of vitiligo. ${ }^{24}$

Westerhof et al performed a left-right comparison study of the combination of fluticasone propionate and UVA versus either fluticasone propionate or UVA for the long-term treatment of vitiligo and concluded that the combination treatment with fluticasone proprionate and UVA is much more effective in reaching complete repigmentation than are fluticasone proprionate and UVA used alone, but large inter-individual differences occurred in the study. ${ }^{25}$

\section{PUVA therapy}

This is the combination of body irradiation with UVA-light and a psoralenderivate. The psoralens most commonly used are 8-methoxypsoralen (8-MOP), 5-methoxypsoralen (5-MOP) and 4,5', 8-trimethylpsoralen (TMP). Already in ancient Egyptian times, plant extracts containing psoralens were used to treat patients with vitiligo. ${ }^{26}$ In the 1940 's, PUVA was introduced to treat vitiligo. ${ }^{27}$ In PUVA therapy, the psoralens can be applied topically or can be taken orally. In vitro studies demonstrated that oral PUVA stimulates melanocytes to proliferate, differentiate and migrate. It also has a local immunomodulatory effect. ${ }^{28}$ Ermis et al performed a placebo-controlled double-blind study to investigate whether the effectiveness of PUVA therapy could be enhanced by combination with topical calcipotriol in the treatment of vitiligo and concluded that concurrent topical calcipotriol potentiates the efficacy of PUVA therapy in the treatment of vitiligo, and that this combination achieves earlier pigmentation with a lower total UVA dosage. ${ }^{29}$

Oral PUVA therapy has a lot of side-effects like nausea, pruritus, phototoxic reactions and a large contrast in pigmentation between normal and repigmented skin. Therefore, PUVA therapy is not the first choice in the treatment of vitiligo.

\section{UVB}

The exact mechanism of vitiligo repigmentation due to UVB is not known. It is probably related to the positive effect of UVB on immunomodulation, growth stimulation and proliferation of melanocytes.

The traditional broadband UVB sources were the first to be used. Broadband UVB therapy, which implies significant exposure to wavelength spectra of less than $300 \mathrm{~nm}$ and even less than $280 \mathrm{~nm}$ (UVC), carries in theory a greater risk of long-term development of nonmelanoma skin cancer compared with narrowband UVB (UVB $311 \mathrm{~nm}$ ), although there is no clinical study that proves this in practice. ${ }^{30}$ 
In 1990, Köster et al reported repigmentation of more than $75 \%$ in 8 of 14 patients suffering from extensive vitiligo, who underwent phototherapy with broadband UVB. Especially the patients with facial lesions of the skin types $\mathrm{V}$ and VI achieved a nearly complete and cosmetically satisfying repigmentation after 12 months of treatment. ${ }^{31}$

Recently, Asawanonda et al performed a small randomized, controlled trial (20 patients) comparing targeted narrowband UVB with targeted broadband UVB and concluded that the clinical responses were similar in both groups. However, the device used in this trial was a targeted UVB device, this implicates that the results cannot be applied to other light sources (eg, excimer laser or total-body irradiation cabinets). ${ }^{32}$

Narrowband UVB (NB-UVB) is a more recent form of phototherapy that uses wavelengths between 305 and $311 \mathrm{~nm}$. Short-time side-effects include pruritus and xerosis, long-term side-effects (eg, carcinogenesis) are unknown. NB-UVB can be used in children, pregnant or lactating women and in individuals with hepatic or kidney dysfunction. ${ }^{23}$

In 1997, Westerhof first reported the use of NB-UVB phototherapy for the treatment of vitiligo and concluded the treatment of patients with vitiligo with 311-nm UV-B radiation is as efficient as with topical PUVA and has fewer adverse effects. ${ }^{33}$ In a recent study by Rath et al narrowband UVB was preferred over broadband UVB. Narrowband UVB and PUVA showed comparable efficacy. ${ }^{22}$

Esfandiarpour et al studied 68 patients with vitiligo. The patients were divided into two groups and treated with NB-UVB in combination with pimecrolimus or with NB-UVB in combination with a placebo cream for 3 months. The authors concluded that, on the face, NB-UVB works better if combined with $1 \%$ pimecrolimus cream rather than used alone. ${ }^{34}$

El Mofty et al performed an evaluation of NB-UVB $311 \mathrm{~nm}$ in the treatment of vitiligo by two independent studies. The first study compared NB-UVB with PUVA therapy, and the second study compared NB-UVB with NB-UVB + psoralens. They showed no statistical difference in repigmentation or side-effects between NB-UVB and PUVA therapy. In the second study, there was no statistical difference in repigmentation between NB-UVB and NB-UVB + psoralens, but the group with psoralens had more side- effects. ${ }^{35}$

In 2007, Yones et al performed a randomized double blind trial in 56 patients with nonsegmental vitiligo, comparing PUVA therapy with NB-UVB therapy and concluded that, in the treatment of nonsegmental vitiligo, NB-UVB therapy is superior to oral PUVA therapy. ${ }^{36}$

Arca et al compared NB-UVB as monotherapy to the combination of NB-UVB + calcipotriol in a randomized study and concluded that NB-UVB is an effective therapy for vitiligo but they could not show an additional effect of topical calcipotriol. ${ }^{37}$

We can conclude that narrowband UVB is as effective (or more effective) than PUVA therapy in the treatment of vitiligo but has fewer side-effects. It is preferred over broadband UVB and is a promising treatment for vitiligo.

\section{Excimer laser}

The xenon chloride excimer laser emits a wavelength of $308 \mathrm{~nm}$. The excimer laser has some useful technical characteristics like an articulated arm and variable spot size. It is possible to selectively turn the beam of light and to treat only the involved area, sparing healthy skin. In vitiligo, this selectivity limits the unwanted hyperpigmentation of non involved skin, which is commonly observed with the other phototherapies. The articulated arm makes it possible to treat body regions that are otherwise difficult to reach (eg, folds and mucosa). Disadvantages include the fact that the limited spot size means that large surfaces $(>20 \%$ of total surface body area) cannot be treated and that purchase and maintenance costs of these devices remain quite expensive. ${ }^{38}$

Hadi et al performed a retrospective chart review of 97 patients (a total of 221 vitiligo patches) with chronic stable vitiligo treated with the excimer laser and concluded that it is an effective and safe modality for the treatment of vitiligo, with good results achieved in a relatively short duration of time. Lesions on the face responded better than lesions elsewhere. ${ }^{39}$

In 2006, Passeron and Ortonne performed a review of 10 studies on the use of the 308 -nm excimer laser for psoriasis and vitiligo. For vitiligo, low fluencies (from 50 to $200 \mathrm{~mJ} / \mathrm{cm}^{2}$ ) were used in 1 to 3 sessions a week for 1 to 6 months, depending on the study. The number of plaques with repigmentation at the end of the treatment was high (57\%-100\%). On average, $20 \%$ to $30 \%$ of treated plaques reach more than $75 \%$ repigmentation, but some series report conflicting results (from $0 \%$ to $75 \%$ ). The clinical response to the treatment is especially dependent of the localization of the lesions. UV-resistant areas (eg, extremities and bony prominences) were more difficult to treat. ${ }^{38}$

Passeron et al examined the efficacy of combined treatment with $0.1 \%$ tacrolimus ointment plus $308 \mathrm{~nm}$ excimer laser versus excimer laser monotherapy. They concluded that the combined therapy was only superior to the excimer laser 
monotherapy for the treatment of UV-resistant vitiliginous lesions. ${ }^{40}$

Goldinger et al did a prospective (left-right) comparative, single blinded study to compare the effectiveness of the excimer laser and the combination of the excimer laser with topical calcipotriol in the treatment of vitiligo. They showed no significant difference in overall repigmentation between the two groups. ${ }^{41}$

Just recently, Sassi et al did a randomized, controlled trial to compare the effectiveness of the $308 \mathrm{~nm}$ excimer laser alone or in combination with topical hydrocortisone 17-butyrate cream in patients with vitiligo, unresponsive to previous treatment with topical steroids or narrowband ultraviolet B phototherapy. They found that recalcitrant vitiligo of the face and neck may benefit from the combination of excimer laser phototherapy with topical hydrocortisone 17-butyrate cream. ${ }^{42}$

The excimer laser is a promising therapy for vitiligo, with good clinical results, especially for the treatment of vitiligo lesions on the face.

\section{Calcipotriol}

Calcipotriol is a vitamin D3 analogue that might be effective on immunomodulatory systems, inflammatory mediators and melanocytes. ${ }^{43}$ Tomita et al showed that vitamin D3 induced features similar to those noted in UV-radiated skin (such as swelling of melanocytes in the epidermis and increased tyrosinase activity resulting in a deposition of melanin in the epidermis). ${ }^{44}$

Kumaran et al conducted a randomized trial that studied the effect of $0,005 \%$ topical calcipotriol, $0.05 \%$ betamethasone dipropionate and their combination in the treatment of localized vitiligo. When used individually, the betamethasone dipropionate and the calcipotriol were found to be equally effective but the combination of the two appeared to give a significantly faster onset of repigmentation along with better stability of the achieved pigmentation and with lesser number of side-effects. ${ }^{45}$

Ameen et al conducted an open study to investigate the efficacy and tolerability of calcipotriol cream as monotherapy and in combination with PUVA therapy. They found that topical calcipotriol appears to be an effective and well-tolerated treatment for vitiligo and can be safely used in combination with PUVA. ${ }^{46}$ Chiavérini et al performed a prospective, right-left comparative, open study and examined the efficiency of topical calcipotriol as a monotherapy for the treatment of vitiligo. They concluded that it was not effective. $^{47}$
However, no randomized controlled trials have been performed yet with calcipotriol as a monotherapy for vitiligo. The combination of calcipotriol and PUVA or UVB has also been studied in other trials, but the results are contradicting as some authors describe a benefit as others do not. ${ }^{41,43}$

\section{Topical immunomodulators (tacrolimus/ pimecrolimus)}

Tacrolimus and pimecrolimus (macrolide immunomodulators) can be used topically and are called topical immunomodulators (TIMs). TIMs inhibit the action of calcineurin, and consequently prevent the transcription of inflammatory cytokines dependent on the transcription factor nuclear factor of activated T-cells (NF-AT). This reversible inhibition of cytokine synthesis affects both $\mathrm{T}$ helper type 1 (Th-1) cytokines (interleukin-2, interferon- $\gamma$ ) and Th-2 cytokines (interleukin-4, interleukin-10). This is considered the working mechanism of action of TIMs in vitiligo. ${ }^{48,49}$

The use of TIMs in vitiligo has been documented in randomized controlled trials and in several case reports and open studies. One randomized controlled study compared $0.1 \%$ tacrolimus and $0.05 \%$ clobetasol cream in 20 children with vitiligo. The level of repigmentation was $49.3 \%$ with clobetasol and $41.3 \%$ with tacrolimus. ${ }^{17}$ Dawid et al performed a double-blind, intra-patient comparison of $1 \%$ pimecrolimus cream with placebo cream in 20 patients with vitiligo predominantly situated on the extremities and not on the face and found no significant change in mean target lesion. ${ }^{49}$

Since 2002, clinical studies have favored the use of calcineurin inhibitors in vitiligo, especially in children, in whom they were shown to be equally effective as corticosteroids. However, in adult patients the effect was mainly restricted to the face and neck, whereas lesions in other regions of the body showed variable responses. ${ }^{50}$ Just recently, Choi et al performed a retrospective review of 79 patients and showed that topical immunomodulators are as effective as topical steroids in repigmentation of vitiligo. They also showed that the duration between start of treatment and onset of repigmentation was significantly shorter in the topical immunomodulator group..$^{51}$

In a prospective placebo-controlled right-left comparison study, Hartmann et al investigated the efficacy and safety of $0.1 \%$ tacrolimus ointment for up to 12 months in 30 adult patients with vitiligo, and tested the influence of additional occlusion. They concluded that $0.1 \%$ tacrolimus ointment proved an effective and safe treatment option for adult patients with vitiligo. Beyond the face and neck areas, repigmentation 
could be achieved only by additional occlusion (eg, household foil, hydrocolloid dressing or polyurethane foil). ${ }^{50}$

A recent randomized, double-blind, placebo-controlled study compared combination of $1 \%$ pimecrolimus with NB-UVB versus placebo with NB-UVB and concluded that, on the face, NB-UVB works better if combined with $1 \%$ pimecrolimus cream than used alone. ${ }^{52}$

Topical calcineurin inhibitors are effective in the treatment of vitiligo, especially when the lesions are situated on the face.

Unlike topical corticosteroids, topical calcineurin inhibitors do not cause skin atrophy. A noted side-effect was a burning sensation at the site of application. In 2005, the Pediatric Advisory Committee of the US FDA implemented a black box warning for tacrolimus and pimecrolimus due to the lack of long-term safety data and the potential risk of the development of malignancies. However, evidence of a causal relation to the development of skin cancer or lymphoma is still missing. More studies are needed to understand this risk. $^{53,54}$

\section{Pseudocatalase cream}

One study showed that patients with vitiligo have low catalase levels in their epidermis in association with accumulation of hydrogen peroxide $\left(\mathrm{H}_{2} \mathrm{O}_{2}\right)$. A topical pseudocatalase cream and calcium used twice daily with UVB therapy, twice weekly, resulted in repigmentation in the majority of cases in a case study with 33 patients after 2-4 months. However, it was not known whether the repigmentation was due to the UVB or the pseudocatalase. ${ }^{55}$ Sanclemente et al compared the effect of topical $0.05 \%$ betamethasone valerate versus catalase/ dismutase superoxide (C/DSO) and concluded that vitiligo repigmentation with topical $\mathrm{C} / \mathrm{DSO}$ at 10 months is similar to repigmentation with topical $0.05 \%$ betamethasone valerate. ${ }^{18}$

More studies on the use of pseudocatalase cream in the treatment of vitiligo are necessary to prove its possible therapeutic effect.

\section{Comparison}

Recently, Lotti et al performed an open-label study comparing the current non-surgical treatments for vitiligo (311-nm narrowband microphototherapy, $0.1 \%$ tacrolimus ointment twice a day, $1 \%$ pimecrolimus cream twice a day, $0.05 \%$ betamethasone dipropionate cream twice a day, $0.005 \%$ calcipotriol ointment twice a day and $10 \% 1$-phenylalanine cream twice a day as a monotherapy or the NB-UVB therapy in combination with the above mentioned local treatments). They concluded that when the single treatments are considered alone, 311-nm narrowband UVB microphototherapy and $0.05 \%$ betamethasone dipropionate cream are the most effective treatments. In combination therapies, $0.05 \%$ betamethasone dipropionate cream plus 311-nm narrowband UVB microphototherapy gave the best repigmentation rate. In this study, the only side-effects registered were cutaneous atrophy with the corticosteroid cream, and stinging and burning with the topical immunomodulators. ${ }^{56}$

\section{Surgical repigmentation therapy}

Surgical repigmentation can be considered in stabilized vitiligo, if other repigmentation therapies fail. All surgical techniques have the same basic principle: to transplant autologous melanocytes from pigmented donor skin to regions without melanocytes. However, patients need to be carefully selected as not every vitiligo patch is suitable for transplantation. Surgical techniques may be considered in stable, non-progressive disease only (there is no consensus on the exact period of stability, various recommendations suggest stable vitiligo for 6 months to 2 years). ${ }^{57}$ Contraindications are a positive Koebner phenomenon and hypertrophic scars or keloids in the past. The site of transplantation is also important, as some areas such as joints, lips, eyelids, genitalia, cutaneous folds, the dorsum of hands and feet, fingers and toes, are difficult to repigment. ${ }^{58}$

There are several methods for surgical repigmentation.

\section{Autologous minipunchgrafting}

The first method is autologous minipunchgrafting were multiple punch biopsies are obtained from a normally pigmented donor site and implanted within achromic areas, separated from each other by 5 to $8 \mathrm{~mm}$. To determine if this technique works for a specific patient, a test-graft is performed (3-5 minigrafts). If pigment has spread from the donor tissue after a period of time and no Koebner phenomenon exists at the donor site, the entire region is grafted. This procedure is useful for limited areas of vitiligo, but a cobblestone appearance, sinking pits, infection or scarring are potential side-effects. ${ }^{3,59}$ In 1988, Falabella treated 22 patients with localized vitiligo with autologous minigrafting and a $90 \%$ to $100 \%$ repigmentation was achieved in 13 patients. ${ }^{60}$ Another study by Sarkar et al showed a repigmentation rate of $40 \%$ to $60 \%$ in the majority of the treated patients who has segmental vitiligo. ${ }^{61}$ Boersma et al performed minigrafts in patients with stable vitiligo vulgaris (stable for 6 months). 14 out of 36 lesions showed $80 \%$ to $99 \%$ repigmentation after 6 months. They state that repigmentation rates of more than $80 \%$ are generally not reached before 6 months after grafting. ${ }^{62}$ 


\section{Epidermal suction blister grafting}

Another technique is epidermal suction blister grafting. Blisters are formed at donor and recipient sites with a suction machine or by freezing with liquid nitrogen for 20 to 25 seconds. The roof of the blister is removed from both sites and the donor graft is placed on top of the denuded recipient site. The grafts are held in place with bandages for 7 days. Repigmentation usually occurs in 1 to 2 weeks, and total repigmentation can occur within 1 to 3 months. ${ }^{3}$ Gupta et al compared epidermal suction blister grafting to punch skin grafting in 49 patients and reported a repigmentation rate of more than $75 \%$ in $67 \%$ of the lesions in the punch graft group and in $82 \%$ of the lesions in the epidermal suction blister group. This difference was not significant. However, the latter technique gives cosmetically better results. ${ }^{63}$ The overall success rate of epidermal suction blister grafting varies between $73 \%$ and $88 \%$ in different studies and is increased if the donor site is treated with PUVA therapy before the procedure. Epidermal grafting shows excellent cosmetic results and is an easy, inexpensive, safe and efficient treatment, however it is a time consuming technique..$^{59}$

\section{Split-thickness grafting}

In split-thickness skin grafting, the whole epidermis and a part of the upper papillary dermis is grafted at the donor site using a hand dermatome or shaving blade. Grafts are positioned on abraded recipient areas that have been prepared previously. The graft is secured with pressure and immobilization. This technique has a success rate of $78 \%$ to $90 \%{ }^{59}$ Ozdemir et al were the first to compare 2 surgical techniques in a blinded matter (epidermal suction blister grafting versus split-thickness skin grafting). In their study, $25 \%$ of patients showed repigmentation with suction blister erosion without grafting, $45 \%$ of patients showed repigmentation with suction blister grafting. Thin split-thickness grafting led to repigmentation in $90 \%$ of patients. ${ }^{64}$ Success rates depend on using a very thin graft to prevent additional cosmetic damage to both recipient and donor areas. Side-effects of this technique are stuck-on effects, persistent hyperpigmentation, milia, perigraft halo of depigmentation and scars on donor areas, and it is not suitable for vitiliginous lesions on the palms, soles, and skin folds. The benefits are the immediate results, the highest success mean rate and the ability to treat difficult areas like eyelids, penis, vagina and lips..$^{65,66}$

\section{Transplantation of non-cultured epidermal cellsuspension}

In 1992, Gauthier and Surleve-Bazeille described a technique in which non-cultured melanocytes were isolated from samples of skin of the hair scalp, then trypsinized and transplanted. The grafting method is carried out in two steps: first, blisters are formed on the depigmented lesions by freezing with liquid nitrogen, then, each blister is injected with a suspension of epidermal cells (mainly keratinocytes and melanocytes). Repigmentation occurred within 25 to 30 days and they concluded that this technique is an effective and simple transplantation method ${ }^{67} \mathrm{~W}$ ith this technique, large areas can be treated (5- to 10-fold the donor area). Disadvantages are a long-lasting erythema (up to 6 months) at the recipient site due to dermabrasion and the need for specialized laboratories with trained personnel. ${ }^{59}$

Van Geel et al performed the first double-blind placebocontrolled study on autologous transplanted epidermal cell suspensions for repigmenting vitiligo. To improve the viscosity and fixation of the cellsuspension, they investigated a modified approach adding hyaluronic acid to the cellular grafting procedure. In this study, transplantation resulted in repigmentation of at least $70 \%$ of the treated area in the majority of patients. They could demonstrate that repigmentation was primarily caused by the transplanted melanocytes, as repigmentation at the placebo treated site was negligible. ${ }^{68}$

Mulekar performed a long-term follow-up study in 142 patients treated with a non-cultured melanocyte-keratinocyte cell transplantation. More than half of the treated patients had a complete repigmentation that was fully retained for a 6-year follow-up period. ${ }^{69}$

\section{Transplantation of in vitro-cultured epidermis}

A shave biopsy of normally pigmented skin is taken. After separating the epidermis from the dermis, the cells are seeded in a medium that allows co-cultivation of melanocytes and keratinocytes. After 3 weeks a cultured sheet is obtained, released by treatment with dispase and attached to a gauze as support. The gauze (to which the epithelium adheres) is placed onto the recipient site after it is treated with dermabrasia. ${ }^{55}$ This technique has the advantage that a large area can be treated (10 fold the donor site). Studies have shown a good repigmentation in $33 \%$ to $54 \%$ of treated patients. ${ }^{59,70,71}$ A side-effect of this technique can be hyperpigmentation, but this tends to disappear after a few months. Another disadvantage is failure of culture and the fact that it is an expensive procedure.

\section{Transplantation of in vitro-cultured melanocytes}

Here, melanocytes are obtained from shave biopsies and cultured in vitro with addition of growth factors and chemical 
Table I Conclusions on treatment for vitiligo

\begin{tabular}{|c|c|c|}
\hline & Conclusion & Remarks \\
\hline \multicolumn{3}{|l|}{ Non-surgical treatments } \\
\hline Topical corticosteroids & $\begin{array}{l}\text { Effective for the treatment of localized } \\
\text { vitiligo }\end{array}$ & Result of meta-analysis \\
\hline Oral corticosteroids & $\begin{array}{l}\text { Have an adjunct value, not very effective } \\
\text { by themselves }\end{array}$ & $\begin{array}{l}\text { No placebo controlled studies have been } \\
\text { performed for oral corticosteroids as a } \\
\text { monotherapy }\end{array}$ \\
\hline (P)UVA & $\begin{array}{l}\text { Broadband UVA alone may have a } \\
\text { therapeutic value PUVA therapy is as } \\
\text { effective as broadband UVB, but with } \\
\text { more side-effects }\end{array}$ & UVA is not studied in RCTs \\
\hline Broadband UVB & $\begin{array}{l}\text { Effective for the treatment for } \\
\text { generalized vitiligo }\end{array}$ & \\
\hline Narrowband UVB & $\begin{array}{l}\text { As effective as broadband UVB, } \\
\text { and sometimes even preferred } \\
\text { over broadband UVB. Preferred for } \\
\text { generalized vitiligo }\end{array}$ & Result of meta-analysis \\
\hline Excimer laser & $\begin{array}{l}\text { Excimer laser is an effective and safe } \\
\text { treatment for vitiligo and gives the best } \\
\text { results if used on the face }\end{array}$ & \\
\hline Calcipotriol & $\begin{array}{l}\text { Contradicting results: some authors } \\
\text { describe a benefit but others do not }\end{array}$ & $\begin{array}{l}\text { No placebo controlled studies have been } \\
\text { performed for topical calcipotriol as a } \\
\text { monotherapy }\end{array}$ \\
\hline Topical immunomodulators & $\begin{array}{l}\text { As effective in repigmentation as topical } \\
\text { corticosteroids }\end{array}$ & $\begin{array}{l}\text { The effect is mainly restricted to the face } \\
\text { and neck }\end{array}$ \\
\hline Pseudocatalase cream & May have an effect & Few studies performed \\
\hline Surgical treatments & & Few (comparative) studies performed \\
\hline Autologous minipunchgrafting & $\begin{array}{l}\text { Success rates of } 40 \%-99 \% \text { (mean success } \\
\text { rate: } 57 \% \text { ) Only a small surface can be } \\
\text { treated }\end{array}$ & $\begin{array}{l}\text { Lower mean success rate than epidermal } \\
\text { suction blister grafting and split-thickness } \\
\text { skin grafting }\end{array}$ \\
\hline Epidermal suction blister grafting & $\begin{array}{l}\text { Success rates of } 73 \%-88 \% \text { (mean success } \\
\text { rate: } 81 \% \text { ) }\end{array}$ & Only a small surface can be treated \\
\hline Split-thickness skin grafting & Success rates of $78 \%-90 \%$ & \\
\hline $\begin{array}{l}\text { Transplantation of non-cultured epidermal } \\
\text { cellsuspension }\end{array}$ & $\begin{array}{l}\text { Success rates of } 59 \%-70 \% \text { (mean success } \\
\text { rate: } 63 \% \text { ) Suitable for larger areas }\end{array}$ & \\
\hline $\begin{array}{l}\text { Transplantation of in vitro-cultured } \\
\text { epidermis }\end{array}$ & Success rates of $33 \%-54 \%$ & \\
\hline $\begin{array}{l}\text { Transplantation of in vitro-cultured } \\
\text { melanocytes }\end{array}$ & Success rates of $22 \%-72 \%$ & \\
\hline \multicolumn{3}{|l|}{ Depigmentation therapy } \\
\hline Monobenzylether of hydroquinone & Effective as a depigmentation therapy & Few studies performed \\
\hline Mequinol & Effective as a depigmentation therapy & Few studies performed \\
\hline \multicolumn{3}{|l|}{ Complementary therapies } \\
\hline L-phenylalanine & May be used as adjuvant to phototherapy & Few studies performed \\
\hline Ginkgo biloba & May be effective as monotherapy & Only shown in one study \\
\hline Polypodium leucomotos & $\begin{array}{l}\text { Can improve narrowband UVB-induced } \\
\text { repigmentation }\end{array}$ & Few studies performed \\
\hline Antioxidants & $\begin{array}{l}\text { Can improve the clinical effectiveness } \\
\text { of narrowband UVB }\end{array}$ & Few studies performed \\
\hline
\end{tabular}

Abbreviations: PUVA, psoralen ultraviolet-A therapy; RCT, randomized controlled trial. 
media. The suspension is then transplanted on abrased recipient areas. Success rates vary between $22 \%$ and $72 \%$. Disadvantages are that it is a time consuming technique and that there are only a few trials published that study this technique. ${ }^{57}$ In 2004, Chen et al performed an analysis of 120 patients with vitiligo who were treated with autologous cultured pure melanocyte suspension combined with carbon dioxide laser abrasion. They concluded that this technique is an effective treatment for patients with stable vitiligo who fail to respond to medical treatments, especially for those with stable localized vitiligo. ${ }^{72}$

As there are no uniform and acceptable criteria for the evaluation of the outcome after surgical repigmentation, it is difficult to compare the results of different studies on different transplantation methods.

In 1998, Njoo et al performed a systematic review of the effectiveness, safety, and applicability of autologous transplantation methods in vitiligo. Recently developed techniques using noncultured epidermal cellsuspension were not included in the review. Split-thickness grafting and epidermal blister grafting were the most effective and safest methods in this review. Minigrafting showed a relatively lower success rate, which could be explained by variations in the size of pigment spread of the punch grafts. They state that postoperative PUVA therapy or exposition to sunlight may improve the repigmentation grade in minigrafting. ${ }^{73}$ However, as no randomized controlled trials were included in the review, results should be interpreted with caution.

\section{Depigmentation therapy}

Depigmentation with monobenzylether of hydroquinone can be considered if vitiligo affects more than $60 \%$ to $80 \%$ of the face or body. The major side-effect is contact or irritant contact dermatitis. Only a few studies (no randomized controlled trials) with hydroquinone for vitiligo have been performed. In a retrospective study of 18 vitiligo patients who used $20 \%$ monobenzylether of hydroquinone as a depigmenting agent, 8 achieved complete depigmentation after 10 months of use and 3 achieved dramatic but no complete depigmentation. ${ }^{74}$ In 1996, Oakley described a rapid repigmentation after depigmentation with monobenzylether of hydroquinone, the mechanism was unknown. ${ }^{75}$

4-methoxyphenol (4-MP) or mequinol is another phenol derivate and has similar melanocytotoxic properties as monobenzylether of hydroquinone. Njoo et al performed a retrospective study on depigmentation therapy in 16 patients and concluded that depigmentation therapy using a 4-MP cream and/or Q-switched ruby laser therapy can be an effective and safe method to remove residual pigment in patients with vitiligo universalis. ${ }^{76}$ However, because there are no randomized controlled trials performed, results should be considered with caution.

\section{Complementary therapies}

Just recently, Szczurko and Boon performed a comprehensive review of literature on different natural health products that may have an effect on vitiligo. The most commonly studied product was L-phenylalanine, not in monotherapy but in combination with phototherapy or other products. Although it was not possible to pool the collected data, the overall outcome was that there is moderate evidence for efficacy of L-phenylalanine as adjuvant to phototherapy. There was no convincing evidence that vitamins have a place in vitiligo treatment. ${ }^{77}$ Ginkgo biloba also seems to be promising as monotherapy for vitiligo in a double-blind, placebo-controlled trial, performed by Parsad et al but more and controlled studies are necessary to confirm this effect. ${ }^{78}$

In 2007, Middelkamp-Hup et al performed a randomized double-blind placebo-controlled study to determine if polypodium leucotomos, an antioxidative and immunomodulatory plant extract, improves narrowband UVB-induced repigmentation. There was a trend towards an increased repigmentation of head and neck (not other body areas) in the group that was treated with the combination of polypodium leucomotos and UVB. This effect was more pronounced in patients with light skin types (type II and III). ${ }^{79}$ Another study by Dell'Anna et al evaluated the combination treatment of antioxidants (containing alpha-lipoid acid, vitamins $\mathrm{C}$ and $\mathrm{E}$ and polyunsaturated fatty acids) and narrowband UVB in a double-blind placebo controlled trial and concluded that the antioxidants significantly improved the clinical effectiveness of narrowband UVB, reducing vitiligo-associated oxidative stress. ${ }^{80}$

\section{Conclusion}

Many studies have been performed to determine which treatment is the best for vitiligo (table 1). Since there is no consensus on the pathogenesis of vitiligo, a treatment to completely cure vitiligo does not exist. More randomized controlled trials on the treatment of vitiligo are necessary.

\section{Disclosures}

The authors have no conflicts of interest to disclose.

\section{References}

1. Bolognia JL, Jorizzo JL, Rapini RP. Dermatology. 2nd Revised edition: Elsevier Health Sciences; 2007. Chapter 65.

2. Taïeb A, Picardo M; VETF Members. The definition and assessment of vitiligo: a consensus report of the Vitiligo European Task Force. Pigment Cell Res. 2007;20(1):27-35. 
3. Kovacs SO. Vitiligo. J Am Acad Dermatol. 1998;38(1):647-666.

4. Le Poole IC, Das PK, van den Wijngaard RM, Bos JD, Westerhof W. Review of the etiopathomechanism of vitiligo: a convergence theory. Exp Dermatol. 1993;2(4):145-153.

5. Oakley AM. Rapid repigmentation after depigmentation therapy: vitiligo treated with monobenzyl ether of hydroquinone. Australas J Dermatol. 1996;37(2):96-98.

6. Spritz RA. The genetics of generalized vitiligo and associated autoimmune diseases. Pigment Cell Res. 2007;20(4):271-278.

7. Passeron T, Ortonne JP. Physiopathology and genetics of vitiligo. J Autoimmun. 2005;25 Suppl:S63-S68.

8. Alkhateeb A, Fain PR, Thody A, Bennett DC, et al. Epidemiology of vitiligo and associated autoimmune diseases in Caucasian probands and their families. Pigment cell res. 2003;16(3):208-214.

9. Gauthier Y, Cario Andre M, Taïeb A. A critical appraisal of vitiligo etiologic theories. Is melanocyte loss a melanocytorrhagy? Pigment Cell Res. 2003;16(4):322-332.

10. Njoo MD, Spuls PI, Bos JD, Westerhof W, et al. Nonsurgical repigmentation therapies in vitiligo. Meta-analysis of the literature. Arch Dermatol. 1998;134(12):1532-1540.

11. Kwinter J, Pelletier J, Khambalia A, Pope E. High-potency steroid use in children with vitiligo: A retrospective study. J Am Acad Dermatol. 2007;56(2):236-241.

12. Schaffer J, Bolognia J. The treatment of hypopigmentation in children. Clinics in Dermatology. 2003;21(4):296-310.

13. Whitton ME, Ashcroft DM, Barrett C, González U. Interventions for vitiligo. Cochrane Database Syst Rev. 2006 Jan 25; (1):CD003263.

14. Bleehen SS. The treatment of vitiligo with topical corticosteroids. Light and electronmicroscopic studies. Br J Dermatol. 1976;94(Suppl 12): S43-S50.

15. Clayton R. A double-blind trial of $0 \%-05 \%$ clobetasol proprionate in the treatment of vitiligo. Br J Dermatol. 1977;96(1):71-73.

16. Kandil E. Treatment of vitiligo with $0-1$ per cent betamethasone 17-valerate in isopropyl alcohol-a double-blind trial. Br J Dermatol. 1974;91(4):457-460.

17. Lepe V, Moncada B, Castanedo-Cazares JP, et al. A double-blind randomized trial of $0.1 \%$ tacrolimus vs $0.05 \%$ clobetasol for the treatment of childhood vitiligo. Arch Dermatol. 2003;139(5):581-585.

18. Sanclemente G, Garcia J, Zuleta J, et al. A double-blind, randomized trial of $0.05 \%$ betamethasone vs. topical catalase/dismutase superoxide in vitiligo. $J$ Eur Acad Dermatol Venereol. In press 2008.

19. Njoo MD, Spuls PI, Bos JD, Westerhof W, et al. Nonsurgical repigmentation therapies in vitiligo. Meta-analysis of the literature. Arch Dermatol. 1998;134(12):1532-1540.

20. Seiter S, Ugurel S, Tilgen W, Reinhold U. Use of high-dose methylprednisolone pulse therapy in patients with progressive and stable vitiligo. Int J Dermatol. 2000;39(8):624-627.

21. Kim SM, Lee HS, Hann SK. The efficacy of low-dose oral corticosteroids in the treatment of vitiligo patients. Int J Dermatol. 1999;38(7): $546-550$.

22. Rath N, Kar HK, Sabhnani S. An open labeled, comparative clinical study on efficacy and tolerability of oral minipulse of steroid (OMP) alone, OMP with PUVA and broad/narrow band UVB phototherapy in progressive vitiligo. Indian J Dermatol Venereol Leprol. 2008;74(4):357-360.

23. Bolognia JL, Jorizzo JL, Rapini RP. Dermatology. 2nd Revised edition: Elsevier Health Sciences; 2007. Chapter 134.

24. El-Mofty M, Mostafa W, Youssef R, et al. Ultraviolet A in vitiligo. Photodermatol Photoimmunol Photomed. 2006;22(4):214-216.

25. Westerhof W, Nieuweboer-Krobotova L, Mulder PG, Glazenburg EJ. Left-right comparison study of the combination of fluticasone propionate and UV-A vs either fluticasone propionate or UV-A alone for the longterm treatment of vitiligo. Arch Dermatol. 1999;135(9):1061-1066.

26. Schneider LA, Hinrichs R, Scharffetter-Kochanek K. Phototherapy and photochemotherapy. Clin Dermatol. 2008;26(5):464-476.

27. El Mofty A. A preliminary clinical report on the treatment of leukoderma with Ammi Majus Linn. J Royal Egypt Med Assoc. 1948;31:651-655.
28. Fitzpatrick TB. Mechanisms of phototherapy of vitiligo. Arch Dermatol. 1997;133(12):1591-1592.

29. Ermis O, Alpsoy E, Cetin L, Yilmaz E. Is the efficacy of psoralen plus ultraviolet A therapy for vitiligo enhanced by concurrent topical calcipotriol? A placebo-controlled double-blind study. Br J Dermatol. 2001;145(3):472-475.

30. Schneider LA, Hinrichs R, Scharffetter-Kochanek K. Phototherapy and photochemotherapy. Clinics in Dermatology. 2008;26(5):464-476.

31. Köster W, Wiskemann A. Phototherapy with UV-B in vitiligo. Zeitschr Hautkr. 1990;65(11):1022-1024.

32. Asawanonda P, Kijluakiat J, Korkij W, Sindhupak W. Targeted broadband ultraviolet $\mathrm{b}$ phototherapy produces similar responses to targeted narrowband ultraviolet B phototherapy for vitiligo: a randomized, double-blind study. Acta Derm Venereol. 2008;88(4):376-381.

33. Westerhof W, Nieuweboer-Krobotova L. Treatment of vitiligo with UV-B radiation vs topical psoralen plus UV-A. Arch Dermatol. 1997;133(12):1525-1528.

34. Esfandiarpour I, Ekhlasi A, Farajzadeh S, Shamsadini S. The efficacy of pimecrolimus $1 \%$ cream plus narrow-band ultraviolet $\mathrm{B}$ in the treatment of vitiligo: A double-blind, placebo-controlled clinical trial. $J$ Dermatolog Treat. In press 2008.

35. El Mofty M, Mostafa W, Esmat S. Narrow band Ultraviolet B $311 \mathrm{~nm}$ in the treatment of vitiligo: two right-left comparison studies. Photodermatol Photoimmunol Photomed. 2006;22(1):6-11.

36. Yones SS, Palmer RA, Garibaldinos TM, Hawk JL. Randomized double-blind trial of treatment of vitiligo: efficacy of psoralen-UV-A therapy vs Narrowband-UV-B therapy. Arch Dermatol. 2007;143(5): $578-584$.

37. Arca E, Tastan HB, Erbil AH, Sezer E, Koc E, Kurumlu Z. Narrow-band ultraviolet $\mathrm{B}$ as monotherapy and in combination with topical calcipotriol in the treatment of vitiligo. $J$ Dermatol. 2006;33(5):338-343.

38. Passeron T, Ortonne JP. Use of the 308-nm excimer laser for psoriasis and vitiligo. Clin Dermatol. 2006;24(1):33-42.

39. Hadi S, Tinio P, Al-Ghaithi K, et al. Treatment of vitiligo using the 308-nm excimer laser. Photomed Laser Surg. 2006;24(3):354-357.

40. Passeron T, Ostovari N, Zakaria W. Topical tacrolimus and the 308-nm excimer laser: a synergistic combination for the treatment of vitiligo. Arch Dermatol. 2004;140(9):1065-1069.

41. Goldinger SM, Dummer R, Schmid P, et al. Combination of 308-nm xenon chloride excimer laser and topical calcipotriol in vitiligo. $J$ Eur Acad Dermatol Venereol. 2007;21(4):504-508.

42. Sassi F, Cazzaniga S, Tessari G, et al. Randomized controlled trial comparing the effectiveness of 308-nm excimer laser alone or in combination with topical hydrocortisone 17-butyrate cream in the treatment of vitiligo of the face and neck. Br J Dermatol. 2008;159(5): $1186-1191$.

43. Goktas EO, Aydin F, Senturk N, Canturk MT, Turanli AY. Combination of narrow band UVB and topical calcipotriol for the treatment of vitiligo. J Eur Acad Dermatol Venereol. 2006;20(5):553-557.

44. Tomita Y, Torinuki W, Tagami H. Stimulation of human melanocytes by vitamin D3 possibly mediates skin pigmentation after sun exposure. J Invest Dermatol. 1988;90(6):882-884.

45. Kumaran MS, Kaur I, Kumar B. Effect of topical calcipotriol, betamethasone dipropionate and their combination in the treatment of localized vitiligo. J Eur Acad Dermatol Venereol. 2006;20(3): 269-273.

46. Ameen M, Exarchou V, Chu AC. Topical calcipotriol as monotherapy and in combination with psoralen plus ultraviolet $\mathrm{A}$ in the treatment of vitiligo. Br J Dermatol. 2001;145:476-479.

47. Chiavérini C, Passeron T, Ortonne JP. Treatment of vitiligo by topical calcipotriol. J Eur Acad Dermatol Venereol. 2002;16(2):137-138.

48. Luger T, Paul T. Potential new indications of topical calcineurin inhibitors. Dermatology. 2007;215 Suppl 1:S45-S54.

49. Dawid M, Veensalu M, Grassberger M, Wolff K. Efficacy and safety of pimecrolimus cream $1 \%$ in adult patients with vitiligo: Results of a randomized, double-blind, vehicle-controlled study. J Dtsch Dermatol Ges. 2006 Nov; 4(11):942-946. 
50. Hartmannv A, Bröcker EB, Hamm H. Occlusive Treatment Enhances Efficacy of Tacrolimus $0.1 \%$ Ointment in Adult Patients with Vitiligo: Results of a Placebocontrolled 12-month Prospective Study. Acta Derm Venereol. 2008;88(5):474-479.

51. Choi CW, Chang SE, Bak H, et al. Topical immunomodulators are effective for treatment of vitiligo. J Dermatol. 2008;35(8):503-507.

52. Esfandiarpour I, Ekhlasi A, Farajzadeh S, Shamsadini S. The efficacy of pimecrolimus $1 \%$ cream plus narrow-band ultraviolet $\mathrm{B}$ in the treatment of vitiligo: A double-blind, placebo-controlled clinical trial. J Dermatolog Treat. 2008;16:1-5.

53. Bieber T, Cork M, Ellis C. Consensus statement on the safety profile of topical calcineurin inhibitors. Dermatology. 2005;211(2):77-78.

54. US Food and Drug Administration. FDA Public Health Advisory: Elidel (pimecrolimus) cream and Protopic (tacrolimus) ointment. 2005 Mar 10. Available from: www.fda.gov/medwatch/SAFETY/2005/safety05. htm\#Elidel.

55. Schallreuter KU, Wood JM, Lemke KR, Levenig C. Treatment of vitiligo with a topical application of pseudocatalase and calcium in combination with short-term UVB exposure: a case study on 33 patients. Dermatology. 1995;190(3):223-229.

56. Lotti T, Buggiani G, Troiano M, et al. Targeted and combination treatments for vitiligo. Comparative evaluation of different current modalities in 458 subjects. Dermatol Ther. 2008;21(Suppl 1):S20-26.

57. Parsad D, Gupta S. Standard guidelines of care for vitiligo surgery. Indian J Dermatol Venereol Leprol. 2008;74 Suppl:S37-S45.

58. Falabella R. Surgical Approaches for Stable Vitiligo. Dermatol Surg. 2006;31(10):1277-1284

59. van Geel N, Ongenae K, Naeyaert JM. Surgical techniques for vitiligo: A review. Dermatology. 2001;202(2):162-166.

60. Falabella R. Treatment of localized vitiligo by autologous minigrafting. Arch Dermatol. 1988;124(11):1649-1655.

61. Sarkar R, Mehta SD, Kanwar AJ. Repigmentation after autologous miniature punch grafting in segmental vitiligo in North Indian patients. J Dermatol. 2001;28(10):540-546.

62. Boersma BR, Westerhof W, Bos JD. Repigmentation in vitiligo vulgaris by autologous minigrafting: results in nineteen patients. $\mathrm{J} \mathrm{Am} \mathrm{Acad}$ Dermatol. 1995;33(6):990-995.

63. Gupta S, Jain VK, Saraswat PK. Suction blister epidermal grafting versus punch skin grafting in recalcitrant and stable vitiligo. Dermatol Surg. 1999;25(12):955-958.

64. Ozdemir M, Cetinkale O, Wolf R et al. Comparison of two surgical approaches for treating vitiligo: a preliminary study. Int $J$ Dermatol. 2002;41(3):135-138.

65. Parsad D, Gupta S. IADVL Dermatosurgery Task Force. Standard guidelines of care for vitiligo surgery. Indian J Dermatol Venereol Leprol. 2008;74 Suppl:S37-S45.
66. Rusfianti M, Wirohadidjodjo YW. Dermatosurgical techniques for repigmentation of vitiligo. Int J Dermatol. 2006;45(4):411-417.

67. Gauthier Y, Surleve-Bazeille JE. Autologous grafting with noncultured melanocytes: A simplified method for treatment of depigmented lesions. J Am Acad Dermatol. 1992;26(2 Patiënt 1):191-194.

68. van Geel N, Ongenae K, De Mil M, Haeghen YV, Vervaet C, Naeyaert JM. Double-blind placebo-controlled study of autologous transplanted epidermal cell suspensions for repigmenting vitiligo. Arch Dermatol. 2004;140(10):1203-1208.

69. Mulekar SV. Long-term follow-up study of 142 patients with vitiligo vulgaris treated by autologous, non-cultured melanocyte-keratinocyte cell transplantation. Int J Dermatol. 2005;44(10):841-845.

70. Andreassi L, Pianigiani E, Andreassi A, Taddeucci P, Biagioli M. A new model of epidermal culture for the surgical treatment of vitiligo. Int $J$ Dermatol. 1998;37(8):595-598.

71. Falabella R, Escobar C, Borrero I. Treatment of refractory and stable vitiligo by transplantation of in vitro cultured epidermal autograft bearing melanocytes. J Am Acad Dermatol. 1992;26(2 Pt 1): 230-236.

72. Chen YF, Yang PY, Hu DN, Kuo FS, Hung CS, Hung CM. Treatment of vitiligo by transplantation of cultured pure melanocyte suspension: analysis of 120 cases. J Am Acad Dermatol. 2004;51(1):68-74.

73. Njoo MD, Westerhof W, Bos JD, Bossuyt PM. A systematic review of autologous transplantation methods in vitiligo. Arch Dermatol. 1998;134(12):1543-1549.

74. Mosher DB, Parrish JA, Fitzpatrick TB. Monobenzylether of hydroquinone. A retrospective study of treatment of 18 vitiligo patients and a review of the literature. Br J Dermatol. 1977;97(6):669-679.

75. Oakley AM. Rapid repigmentation after depigmentation therapy: vitiligo treated with monobenzyl ether of hydroquinone. Australas $J$ Dermatol. 1996 May;37(2):96-98.

76. Njoo MD, Vodegel RM, Westerhof W. Depigmentation therapy in vitiligo universalis with topical 4-methoxyphenol and the Q-switched ruby laser. J Am Acad Dermatol. 2000;42(5 Pt 1):760-769.

77. Szczurko O, Boon HS. A systematic review of natural health product treatment for vitiligo. BMC Dermatology. 2008;22(8):2.

78. Parsad D, Pandhi R, Juneja A. Effectiveness of oral Ginkgo biloba in treating limited, slowly spreading vitiligo. Clin Exp Dermatol. 2003;28(3):285-287.

79. Middelkamp-Hup MA, Bos JD, Rius-Diaz F, Gonzalez S, Westerhof W. Treatment of vitiligo vulgaris with narrow-band UVB and oral Polypodium leucotomos extract: a randomized double-blind placebo-controlled study. J Eur Acad Dermatol Venereol. 2007;21(7):942-950.

80. Dell'Anna ML, Mastrofrancesco A, Sala R, et al. Antioxidants and narrow band-UVB in the treatment of vitiligo: a double-blind placebo controlled trial. Clin Exp Dermatol. 2007;32(6):631-636. 
